\title{
Alternative Approach for the Calculation of Magnetic Field due to Magnet for Magnetic Field Visualization and Evaluation
}

\author{
Kishor Kaphle, Gyanendra Karki, Amrit Panthi \\ Department of Electrical Engineering, Pulchowk Campus \\ Institute of Engineering, Tribhuvan University, Kathmandu, Nepal \\ Corresponding author:071bel320@pcampus.edu.np
}

Received: Sep 28, $2018 \quad$ Revised: Dec 9, $2018 \quad$ Accepted: Dec 15, 2018

\begin{abstract}
The magnetic field of different geometry of the permanent magnet is analytically calculated by using basic principles of the magnetism in very easier approach. Concept of origin shifting and geometrical shape transformation are used to formulate the formula for cuboidal, cubical and cylindrical permanent magnets. This concept can be used for the analysis of magnetic field distribution in space around for permanent magnet as well as electromagnet in a very easier approach. Handy and simplified software is made to calculate the magnetic field due to permanent magnet and electromagnet at any desired position on space. Magnetic field visualization is also done in both magnitude and direction by using MATLAB.
\end{abstract}

Key words: Magnetic moment, Scalar potential, Vector potential, Helmholtz equation, and Magnetic field.

\section{Introduction}

In ancient times, magnetism was believed to be the magical force. With the transition of time, many researches and scientific breakthrough has taken place. At this date, we are very much familiar with that magical force. The spin and the orbital motion in an atom causes the magnetism but there are plenty to be explored and discovered about the cause and property of magnetism. Numerous effort has been made to unify the Gravitational and magnetic force with the references that both possess the unusual attraction force. Electricity and magnetism are inseparable quantity. The source of magnetism is partly the rotation of magnet and partly revolution around the nucleus. The unfilled electron in the atom give rise to magnetism. A filled atomic shell has equal number of electrons consisting up and down spin which results in zero magnetic field. On the other hand, an unfilled shell, results in small magnetization from the orbiting electrons which in turn results in a non-zero net magnetic field of the atom. Earth is a giant magnet. It is not possible for the earth to cause magnetism with the same principle of artificial or man-made permanent magnet. Due to the presence of the tremendous temperature 
inside the core where the existence state of permanent magnetism is not possible. It is believed that the cause of Earth magnetism is dynamo effect in the earth core. Dynamo needs source to drive it otherwise it would decay on a time scale of 200,000 years or so. There are many unsolved mysteries in this field including gravitational setting, tidal forces, buoyance of lighter element and their radioactivity. In order to exist current in the core of earth, it requires molten moving which is ensured by the rotation of Earth [8] [10]. Magnetism is inseparable part of technology. There is numerous application of the permanent magnet such as speaker, electric motors, relay switches, solenoid engines, memory storage, MRI scanner, smart phones, small fridge magnets, etc. Magnetic strength and magnetic field is the governing term of magnetism. It is well known to all; the magnetic field has the curved path but visualizing that shape is a difficult task. Calculating precise value of magnetic field at desired position is necessary in order to accomplish the project related to magnetism. Cyclotron and most of the particle accelerator uses magnetic field to accelerate the particles.

There are many methods for the calculation of magnetic field produce by the permanent magnet, which uses the numerical approximations using Legendre polynomial, greens function, Fourier expansion, hypergeometric series [1], Azimuthal geometry [9], Columbian approach [5], Gauss law [4], etc. In this paper, easier approach for the computation of the magnetic field due to permanent magnet by using triple integration, volume transformation for cylindrical magnet in the cartesian co-ordinate system is chosen. This formula could even be used for the electromagnet in order to calculate the magnitude and direction of magnetic field at any desired point in space around it. Throughout the paper, it is tried to keep things simpler and easier.

\section{Magnetic Moment and Vector Potential}

Magnetic moment $(\mathrm{m})$ gives the value of torque which a magnetic dipole will experience when placed in an external magnetic field. Magnetic moment can be considered as quantity having both magnitude and direction. Magnetic moment is directly proportional to the magnetic field produce by the magnet.

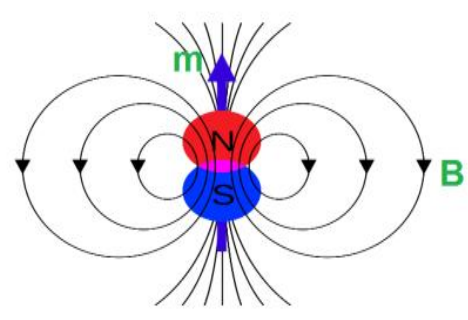

Fig.1: Direction of Magnetic moment and magnetic field
The direction of the magnetic moment is from the south to North Pole inside the magnet whereas it is from north to South Pole outside the magnet. In short, it can be stated that magnetic moment refers to system's magnetic dipole moment [6]. The scalar and vector potential of magnetic field with magnetic moment $m$ at any distance $r$ is given by the following formulae:

$$
\emptyset(r)=\frac{1}{4 \pi} \int_{\text {all space }} \frac{\nabla^{\prime} \cdot F\left(r^{\prime}\right)}{\left|r-r^{\prime}\right|} d v^{\prime}
$$

$$
A(r)=\frac{1}{4 \pi} \int_{\text {all space }} \frac{\nabla^{\prime} \times F\left(r^{\prime}\right)}{\left|r-r^{\prime}\right|} d v^{\prime}
$$


If the gradient of scalar potential and curl of vector potential are known then its vector function can be computed. According to Helmholtz decomposition equation vector function is given:

$\vec{F}=-\nabla \phi+\nabla \times \vec{A}$, where $\emptyset$ and A represent scalar potential and vector potential respectively.

Helmholtz decomposition equation is the foundation in the mathematics, physics, and in the area of vector calculus. According to Helmholtz decomposition theory any sufficiently smooth, rapidly decaying vector field in three dimensions can be resolved into the sum of a solenoidal vector field and an irrotational vector field, which is commonly known as the Helmholtz decomposition equation. It is also called as Helmholtz representation equation. Irrotational means curl zero and solenoidal means divergence zero vectors. According to Helmholtz decomposition, a vector field satisfying appropriate decay and smoothness criteria can be expressed in the form $\vec{F}=-\nabla \phi+\nabla \times \vec{A}$ where $\phi$ is a scalar field, called scalar potential, and A is a vector field, called a vector potential.

$$
\vec{A}(r)=\frac{\mu O}{4 \Pi} \frac{\vec{m} \times \vec{r}}{|\vec{r}|^{3}}
$$

For a permanent magnet, $d m=M d v$, where, $\mathrm{M}$ is magnetization $\mathrm{dv}$ is the differential volume. For a magnet divergence of a vector function is zero. So, it implies that there is no scalar potential ' $\phi$ ' in case of permanent magnetism $[3,11]$. Hence, it is left with the curl of vector potential only.

$$
\vec{B}(r)=\nabla \times \vec{A} \quad \vec{B}(r)=\frac{\mu o}{4 \Pi}\left(\frac{3 \vec{r}(\vec{m} \cdot \vec{r})}{|\vec{r}|^{5}}-\frac{\vec{m}}{|\vec{r}|^{3}}\right)
$$

\section{Magnetic Field Due to Cuboid}

Considering the elementary volume, the formula of $\mathrm{B}(\mathrm{r})$ was integrated for whole volume of cuboid. The direction of the magnetic moment is taken upward. It should be noted that the

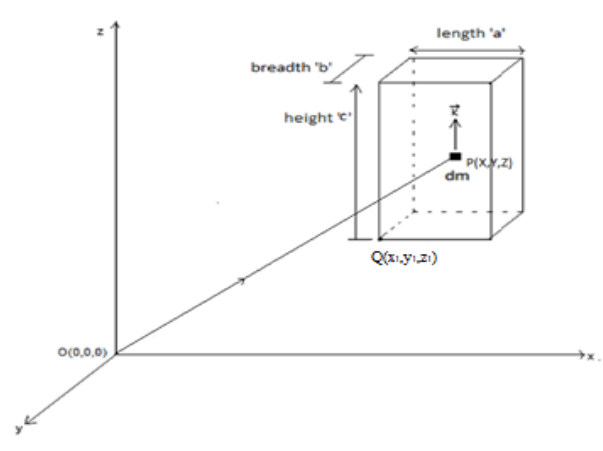

Fig. 2: Permanent magnet place at arbitrary point on space direction of $\mathrm{m}$ is taken upward assuming the magnet is placed as shown in the figure below. It will also be correct when the magnet is placed upside down as in the figure below.

Distance between origin and point $\mathrm{P}$ is: $r=\sqrt{x^{2}+y^{2}+z^{2}}$

For elementary volume $\mathrm{dv}$ with dipole moment $\mathrm{dm}$, 
$d \vec{B}(r)=\frac{\mu o}{4 \Pi}\left(\frac{3(x \vec{i}+y \vec{j}+z \vec{k})(d m \vec{k} \cdot(x \vec{i}+y \vec{j}+z \vec{k}))}{|x \vec{i}+y \vec{j}+z \vec{k}|^{\frac{5}{2}}}-\frac{d m \vec{k}}{|x \vec{i}+y \vec{j}+z \vec{k}|^{\frac{3}{2}}}\right)$

For entire volume, taking triple integration,

$\vec{B}(r)=\frac{\mu o M}{4 \Pi} \int_{x_{1}}^{\left(x_{1}+a\right)} \int_{y_{1}}^{\left(y_{1}+b\right)} \int_{z_{1}}^{\left(z_{1}+c\right)}\left(\frac{3 x z}{r^{\frac{5}{2}}} \vec{i}+\frac{3 y z}{r^{\frac{5}{2}}} \vec{j}+\frac{\left(2 z^{2}-x^{2}-y^{2}\right)}{r^{\frac{5}{2}}} \vec{k}\right) d x d y d z$

The integration was carried out using MATLAB and Mathematica.

$\vec{B}(r)=\frac{\mu o M}{4 \Pi}\left[\left[\left[\left[\left(\log (y+r) \vec{i}+\log (x+r) \vec{j}-\tan ^{-1}\left(\frac{x y}{z r}\right) \vec{k}\right)\right]_{x_{1}}^{x_{1}+a}\right]_{y_{1}}^{y_{1}+b}\right]_{z_{1}}^{z_{1}+c}\right]$

Assumptions:

$$
\begin{aligned}
& r=\sqrt{x_{1}^{2}+y_{1}^{2}+z_{1}^{2}} \quad r_{1}=\sqrt{\left(x_{1}+a\right)^{2}+y_{1}^{2}+z_{1}^{2}} \\
& r_{2}=\sqrt{x_{1}^{2}+\left(y_{1}+b\right)^{2}+z_{1}^{2}} \quad r_{13}=\sqrt{\left(x_{1}+a\right)^{2}+y_{1}^{2}+\left(z_{1}+c\right)^{2}} \\
& r_{3}=\sqrt{x_{1}^{2}+y_{1}^{2}+\left(z_{1}+c\right)^{2}} \quad r_{23}=\sqrt{x_{1}^{2}+\left(y_{1}+b\right)^{2}+\left(z_{1}+c\right)^{2}} \\
& r_{12}=\sqrt{\left(x_{1}+a\right)^{2}+\left(y_{1}+b\right)^{2}+z_{1}^{2}} \quad r_{123}=\sqrt{\left(x_{1}+a\right)^{2}+\left(y_{1}+b\right)^{2}+\left(z_{1}+c\right)^{2}} \\
& B_{x_{1}}=\left[\frac{\mu o M}{4 \Pi} \log \left(\frac{\left(y_{1}+b+r_{23}\right)\left(y_{1}+b+r_{12}\right)\left(y_{1}+r_{13}\right)\left(y_{1}+r\right)}{\left(y_{1}+b+r_{2}\right)\left(y_{1}+b+r_{123}\right)\left(y_{1}+r_{1}\right)\left(y_{1}+r_{3}\right)}\right]\right. \\
& B_{y_{1}}=\left[\frac{\mu o M}{4 \Pi} \log \left(\frac{\left(x_{1}+a+r_{12}\right)\left(x_{1}+a+r_{13}\right)\left(x_{1}+r_{23}\right)\left(x_{1}+r\right)}{\left(x_{1}+a+r_{123}\right)\left(x_{1}+a+r_{1}\right)\left(x_{1}+r_{2}\right)\left(x_{1}+r_{3}\right)}\right)\right] \\
& B_{z_{1}}=\left[\frac { \mu o M } { 4 \Pi } \left[-\tan ^{-1}\left(\frac{\left(x_{1}+a\right)\left(y_{1}+b\right)}{r_{123}\left(z_{1}+c\right)}\right)+\tan ^{-1}\left(\frac{x_{1}\left(y_{1}+b\right)}{r_{23}\left(z_{1}+c\right)}\right)+\tan ^{-1}\left(\frac{y_{1}\left(x_{1}+a\right)}{r_{13}\left(z_{1}+c\right)}\right)-\tan ^{-1}\left(\frac{x_{1} y_{1}}{r_{123}\left(z_{1}+c\right)}\right)+\right.\right. \\
& \left.\tan ^{-1}\left(\frac{\left(x_{1}+a\right)\left(y_{1}+b\right)}{r_{123} z_{1}}\right)-\tan ^{-1}\left(\frac{x_{1}\left(y_{1}+b\right)}{r_{2} z_{1}}\right)-\tan ^{-1}\left(\frac{\left(x_{1}+a\right) y_{1}}{r_{1}\left(z_{1}+c\right)}\right)+\tan ^{-1}\left(\frac{x_{1} y_{1}}{r z_{1}}\right)\right] \\
& \vec{B}=B_{x_{1}} \vec{i}+B_{y_{1}} \vec{j}+B_{z_{1}} \vec{k}
\end{aligned}
$$

\section{Comparison and Validity}

The Formula for the magnetic field on the symmetry axis of an axially magnetized cylinder magnet (disc or rod), which are commonly found in many text books of magnetism is [2] [11]

$$
B=\frac{B_{r}}{2}\left(\frac{(D+Z)}{\sqrt{R^{2}+(D+Z)^{2}}}-\frac{Z}{\sqrt{R^{2}+Z^{2}}}\right)
$$




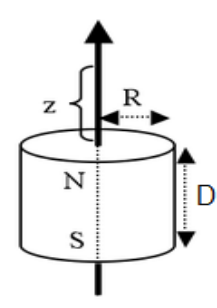

Fig. 3: Permanent magnet placed at $\mathrm{Z}$-axis

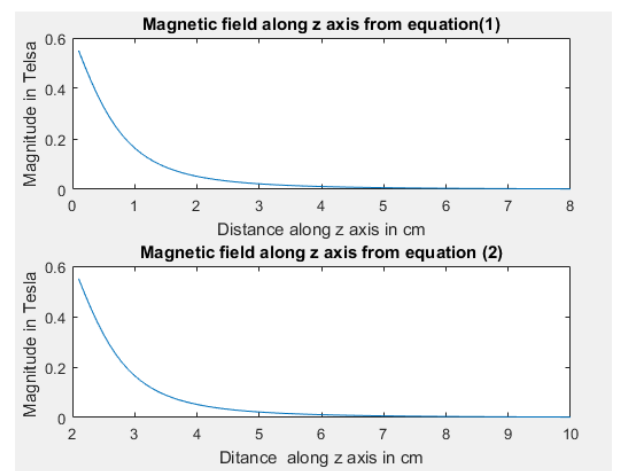

Fig. 4: Comparison of magnitude of field among equation 1 and equation 2 along $z$ axis only
$\mathbf{B}_{\mathbf{r}}$ : Remanence field, independent of the magnet's geometry

$\mathbf{Z}$ : Distance from a pole face on the symmetrical axis

D: Thickness (or height) of the cylinder

R: Semi-diameter (radius) of the cylinder

The unit of length can be selected arbitrarily, as long as it is the same for all lengths.

Dimension during comparison

Cylinder:

$\operatorname{radius}(\mathrm{r})=1 \mathrm{~cm}$

height $(\mathrm{h})=2 \mathrm{~cm}$

Cuboid:

Equivalent dimensions of area: $\pi \mathrm{r}^{2}=(\mathrm{ab})$

$\mathrm{a}=-1.772 \mathrm{~cm}$

$\mathrm{b}=-1.772 \mathrm{~cm}$

$\mathrm{c}=2 \mathrm{~cm}$

\section{Shape Transformation for Cylindrical Magnet into Equivalent Square Faced Magnet}

Comparing the value of magnetic field obtain from equation (1) and equation (2) along $\mathrm{z}$ axis by a cylindrical magnet. Fig. 4, shows that the validity of shape transformation. The above curves show that there is not significant change in value of magnetic field along $\mathrm{z}$ axis for both cylindrical magnet and equivalent transformed cuboidal magnet. The curve obtain from both was almost similar and of maximum of $-1.16 \%$ error at a point $(0,0,0.9)$, which means the maximum error in the value of magnetic field is obtained at a distance of $0.9 \mathrm{~cm}$ from nearest central point of magnet.

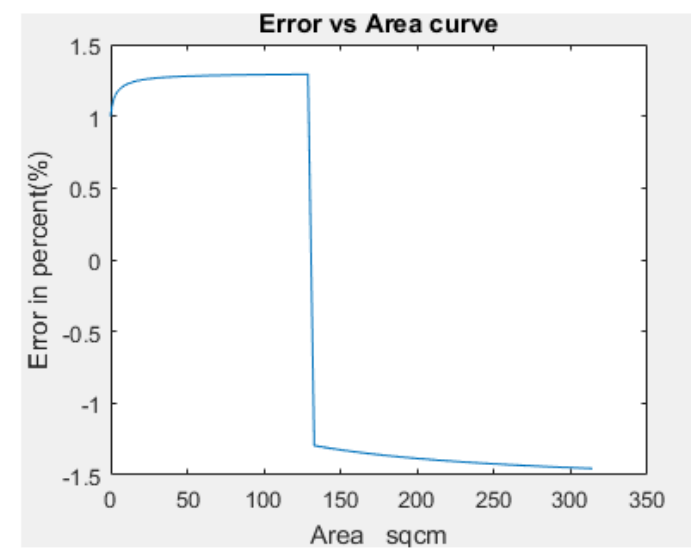

Fig. 5: Compensating area vs. error
The above figure- 5 shows the variations in error for different dimensions during shape transformation. It also shows that initially the error is almost consistent (1.25\%) but as the area of cylinder (to be transformed in equivalent cuboid) goes on increasing there is sharp transition in nature of error after an area of 128.6796 sq. $\mathrm{cm}$ and on further increasing the error again become almost consistent.

Note: (radius was taken from 0.1 to $10 \mathrm{~cm}$ ) 


\section{Derivation for Solenoid}

The derived formula for Permanent Magnet is derived for the solenoid by replacing: $M=N^{*} I$, where

$\mathrm{M}=$ Magnetization, $\quad \mathrm{N}=$ Number of turns, $\quad \mathrm{I}=$ Current through turn

\section{Validity of Derived Formula for Solenoid}

The common formula for calculation of magnetic field of solenoid along axis is given by:

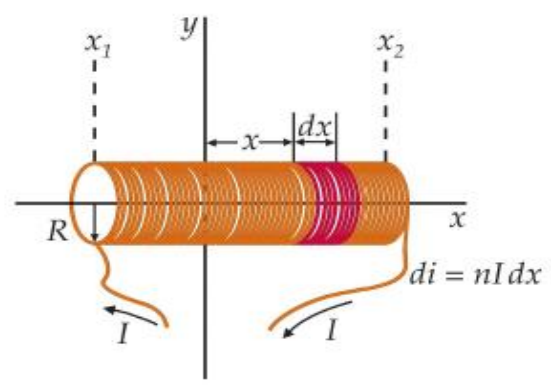

$$
\begin{aligned}
& B_{x}=\frac{\mu o n I}{2} R^{2} \int_{x_{1}}^{x_{2}} \frac{d x^{\prime}}{\left(\left(x-x^{\prime}\right)^{2}+R^{2}\right)^{\frac{3}{2}}} \\
& B_{x}=\frac{\mu o n I}{2}\left(\frac{\left(x-x_{1}\right)}{\sqrt{\left(x-x_{1}\right)^{2}+R^{2}}}-\frac{\left(x-x_{2}\right)}{\sqrt{\left(x-x_{2}\right)^{2}+R^{2}}}\right)
\end{aligned}
$$

\section{Fig. 6: Solenoid placed along $x$-axis with n number of turns and I current}

Comparing the value of magnetic field obtain from equation (1) and equation (3) along axis as shown in figure by solenoid. Fig. 7 shows that the validity of shape transformation.

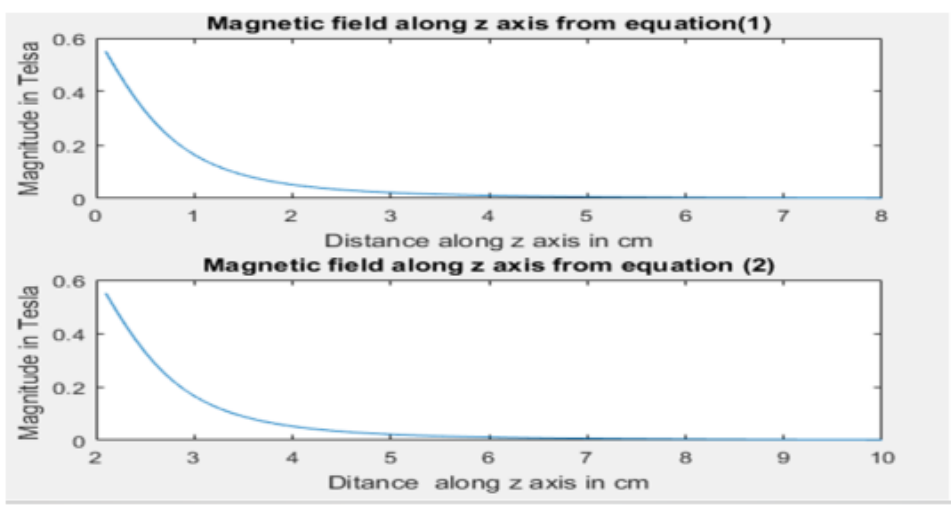

Fig.7: Comparison of equation (1) with equation (3)

The above curves show that there is not significant change in value of magnetic field along $\mathrm{z}$ axis for both equivalent transformed cuboidal (originally cylindrical) magnet and solenoid. The curve obtain from both, was almost similar and of maximum of $-1.16 \%$ error at a point $(0,0,0.9)$. This also suggests that the formula gives nearly accurate result. 


\section{Derivation of Magnetic Field Equation at Space by Keeping}

\section{Center of Magnet at Origin}

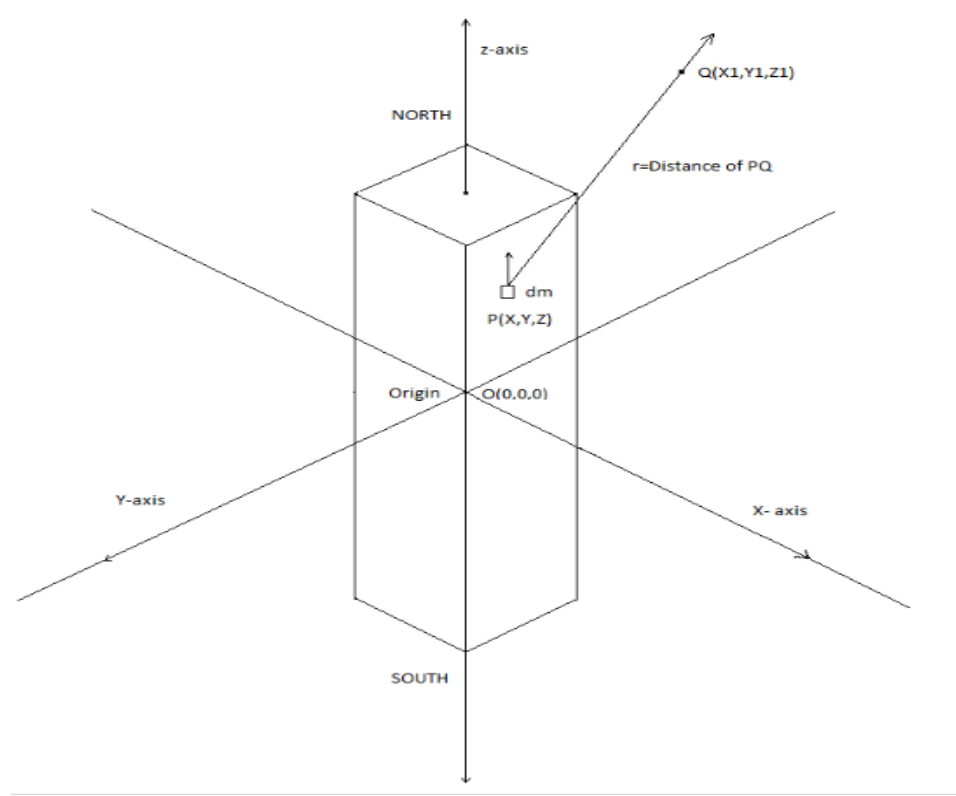

Fig. 8: Permanent magnet with its center placed at origin

Some assumptions for simplicity:

$$
\begin{array}{lll}
r_{11}=\sqrt{\left(x_{1}-\frac{a}{2}\right)^{2}+\left(y_{1}-\frac{b}{2}\right)^{2}+\left(z_{1}-\frac{c}{2}\right)^{2}} & r_{18}=\sqrt{\left(x_{1}+\frac{a}{2}\right)^{2}+\left(y_{1}+\frac{b}{2}\right)^{2}+\left(z_{1}+\frac{c}{2}\right)^{2}} \\
r_{12}=\sqrt{\left(x_{1}+\frac{a}{2}\right)^{2}+\left(y_{1}+\frac{b}{2}\right)^{2}+\left(z_{1}-\frac{c}{2}\right)^{2}} & r_{21}=\sqrt{\left(x_{1}-\frac{a}{2}\right)^{2}+\left(y_{1}-\frac{b}{2}\right)^{2}+\left(z_{1}-\frac{c}{2}\right)^{2}} \\
r_{13}=\sqrt{\left(x_{1}+\frac{a}{2}\right)^{2}+\left(y_{1}-\frac{b}{2}\right)^{2}+\left(z_{1}+\frac{c}{2}\right)^{2}} & r_{22}=\sqrt{\left(x_{1}+\frac{a}{2}\right)^{2}+\left(y_{1}-\frac{b}{2}\right)^{2}+\left(z_{1}-\frac{c}{2}\right)^{2}} \\
r_{14}=\sqrt{\left(x_{1}-\frac{a}{2}\right)^{2}+\left(y_{1}+\frac{b}{2}\right)^{2}+\left(z_{1}+\frac{c}{2}\right)^{2}} & r_{23}=\sqrt{\left(x_{1}-\frac{a}{2}\right)^{2}+\left(y_{1}-\frac{b}{2}\right)^{2}+\left(z_{1}-\frac{c}{2}\right)^{2}} \\
r_{15}=\sqrt{\left(x_{1}+\frac{a}{2}\right)^{2}+\left(y_{1}-\frac{b}{2}\right)^{2}+\left(z_{1}-\frac{c}{2}\right)^{2}} & r_{24}=\sqrt{\left(x_{1}+\frac{a}{2}\right)^{2}+\left(y_{1}+\frac{b}{2}\right)^{2}+\left(z_{1}-\frac{c}{2}\right)^{2}} \\
r_{16}=\sqrt{\left(x_{1}-\frac{a}{2}\right)^{2}+\left(y_{1}+\frac{b}{2}\right)^{2}+\left(z_{1}-\frac{c}{2}\right)^{2}} & r_{25}=\sqrt{\left(x_{1}-\frac{a}{2}\right)^{2}+\left(y_{1}-\frac{b}{2}\right)^{2}+\left(z_{1}+\frac{c}{2}\right)^{2}} \\
r_{17}=\sqrt{\left(x_{1}-\frac{a}{2}\right)^{2}+\left(y_{1}-\frac{b}{2}\right)^{2}+\left(z_{1}+\frac{c}{2}\right)^{2}} & r_{26}=\sqrt{\left(x_{1}+\frac{a}{2}\right)^{2}+\left(y_{1}-\frac{b}{2}\right)^{2}+\left(z_{1}+\frac{c}{2}\right)^{2}}
\end{array}
$$




$$
\begin{aligned}
& r_{27}=\sqrt{\left(x_{1}+\frac{a}{2}\right)^{2}+\left(y_{1}-\frac{b}{2}\right)^{2}+\left(z_{1}+\frac{c}{2}\right)^{2}} \quad r_{28}=\sqrt{\left(x_{1}-\frac{a}{2}\right)^{2}+\left(y_{1}+\frac{b}{2}\right)^{2}+\left(z_{1}+\frac{c}{2}\right)^{2}} \\
& B_{x}=\frac{\mu o M}{4 \Pi} \log \left(\frac{\left(\frac{b}{2}-y_{1}+r_{11}\right)\left(-\frac{b}{2}-y_{1}+r_{12}\right)\left(\frac{b}{2}-y_{1}+r_{13}\right)\left(-\frac{b}{2}-y_{1}+r_{14}\right)}{\left.\left(\frac{b}{2}-y_{1}+r_{15}\right)\left(-\frac{b}{2}-y_{1}+r_{16}\right)\left(\frac{b}{2}-y_{1}+r_{17}\right)\left(-\frac{b}{2}-y_{1}+r_{18}\right)\right]}\right. \\
& B_{y}=\frac{\mu o M}{4 \Pi} \log \left(\frac{\left(-\frac{a}{2}-x_{1}+r_{21}\right)\left(\frac{a}{2}-x_{1}+r_{22}\right)\left(\frac{a}{2}-x_{1}+r_{23}\right)\left(-\frac{a}{2}-x_{1}+r_{24}\right)}{\left(-\frac{a}{2}-x_{1}+r_{25}\right)\left(\frac{a}{2}-x_{1}+r_{26}\right)\left(\frac{a}{2}-x_{1}+r_{27}\right)\left(-\frac{a}{2}-x_{1}+r_{28}\right)}\right) \\
& B_{z}=\frac{\mu o M}{4 \Pi}\left[\tan ^{-1}\left(\frac{\left(\frac{a}{2}-x_{1}\right)\left(\frac{b}{2}-y_{1}\right)}{\left(\frac{c}{2}-z_{1}\right) r_{31}}\right)+\tan ^{-1}\left(\frac{\left(-\frac{a}{2}-x_{1}\right)\left(\frac{b}{2}-y_{1}\right)}{\left(\frac{c}{2}-z_{1}\right) r_{32}}\right)+\tan ^{-1}\left(\frac{\left(\frac{a}{2}-x_{1}\right)\left(\frac{b}{2}-y_{1}\right)}{\left(\frac{c}{2}-z_{1}\right) r_{33}}\right)-\tan ^{-1}\left(\frac{\left(-\frac{a}{2}-x_{1}\right)\left(-\frac{b}{2}-y_{1}\right)}{\left(\frac{c}{2}-z_{1}\right) r_{34}}\right)\right. \\
& \left.+\tan ^{-1}\left(\frac{\left(\frac{a}{2}-x_{1}\right)\left(\frac{b}{2}-y_{1}\right)}{\left(-\frac{c}{2}-z_{1}\right) r_{35}}\right)-\tan ^{-1}\left(\frac{\left(-\frac{a}{2}-x_{1}\right)\left(\frac{b}{2}-y_{1}\right)}{\left(-\frac{c}{2}-z_{1}\right) r_{36}}\right)-\tan ^{-1}\left(\frac{\left(-\frac{a}{2}-x_{1}\right)\left(\frac{b}{2}-y_{1}\right)}{\left(-\frac{c}{2}-z_{1}\right) r_{37}}\right)-\tan ^{-1}\left(\frac{\left(\frac{a}{2}-x_{1}\right)\left(-\frac{b}{2}-y_{1}\right)}{\left(-\frac{c}{2}-z_{1}\right) r_{38}}\right)\right] \\
& \overrightarrow{B(r)}=\overrightarrow{B_{x}+\vec{B}_{y}+\overrightarrow{B_{z}}}
\end{aligned}
$$

\section{Magnetic Field Visualizations}

In order to visualize the magnetic field more precisely the formula is derived for $2 \mathrm{D}$ with same approach as followed in above derivation. The final result was stream plotted using MUPAD in MATLAB. Square plane magnet with dimension Length $(1)=0.5 \mathrm{~cm}$ and Breadth $(\mathrm{b})=0.5 \mathrm{~cm}$ is used.

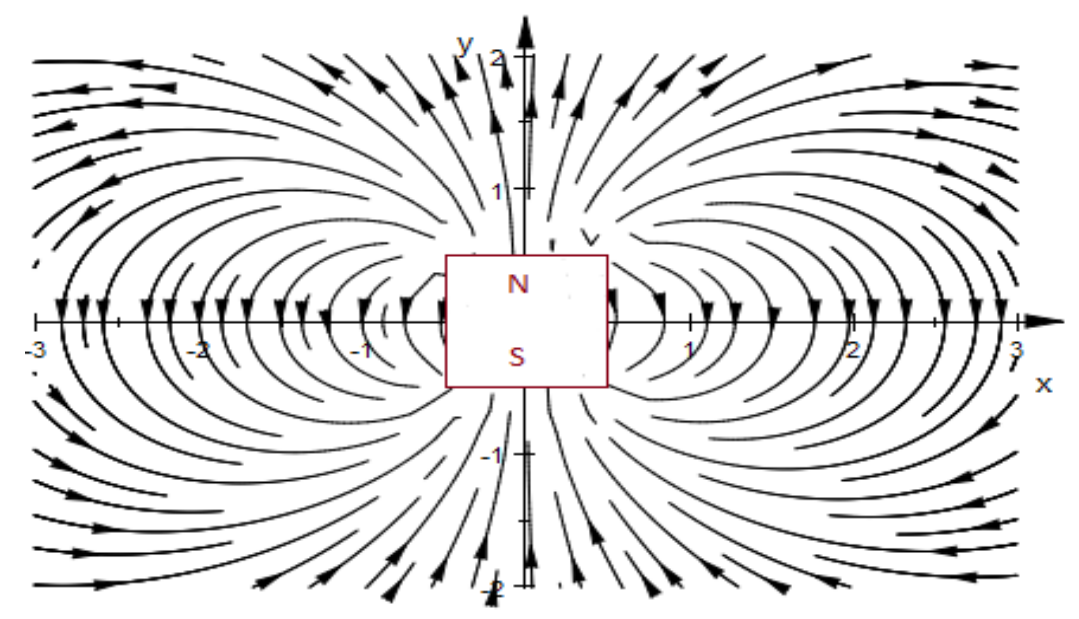

Fig. 9: Showing magnetic field using stream plot MUPAD in MATLAB 
The derived equation of magnetic field was plotted using scatter plot keeping $x=2$ in equation 2 .

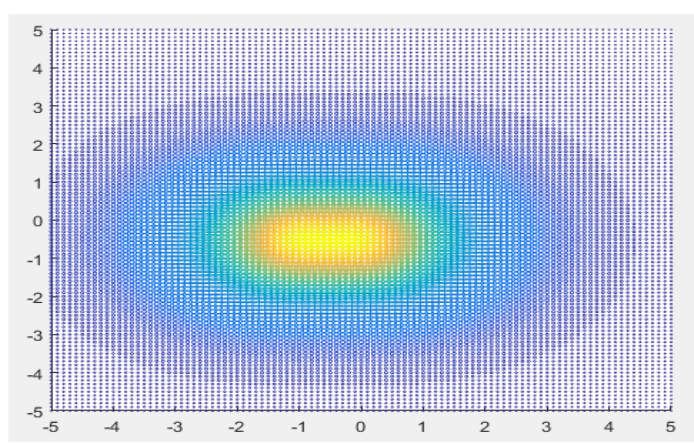

In Fig. 8, the vector lines show the magnitude and direction of magnetic field around the magnet in the $2 \mathrm{D}$ space. In figure, the variation in density of scattered bubbles shows the magnitude distribution of magnetic field in the 2D space.

Fig. 10: Showing magnitude of magnetic field using scatter plot MATLAB

\section{Magnetic Field Visualization Using MATLAB for Attraction and Repulsion of Two Magnets}

The field visualization for attraction of two magnets by derived formula is shown below.

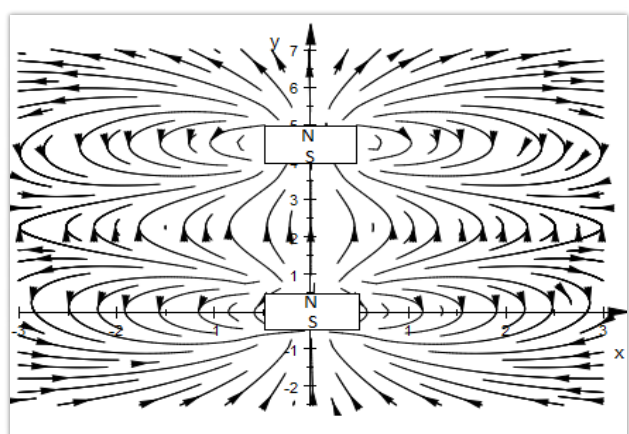

Fig. 11: Field visualization for attraction of two magnets
The magnetic line of force directing from south pole of one magnet to north pole of another magnet, which verifies the attraction of two magnets.

Note: Square plane magnets with dimension Length $(\mathrm{l})=0.5 \mathrm{~cm}$ and Breadth $(\mathrm{b})=0.5 \mathrm{~cm}$. Magnets lies between $-0.5 \mathrm{~cm}$ to $0.5 \mathrm{~cm}$ and $4 \mathrm{~cm}$ to $5 \mathrm{~cm}$ along $\mathrm{z}$ axis and between $-0.5 \mathrm{~cm}$ to $0.5 \mathrm{~cm}$ along $\mathrm{x}$ axis.

The field visualization for repulsion of two magnets by derived formula is shown below.

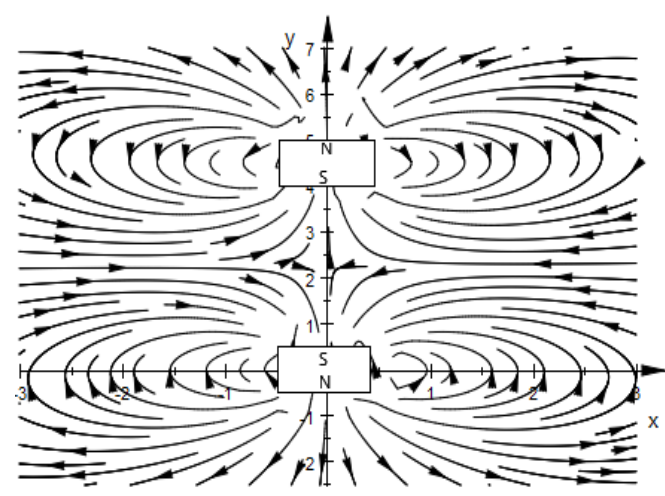
The magnetic line of force directing from south pole of one magnet is reflected by south pole of another magnet, which verifies the repulsion of two magnets. From data, the co-ordinates for the formation of magnetic vortex could also be determined. Note: Square plane magnets with dimension Length $(1)=0.5 \mathrm{~cm}$ and Breadth $(\mathrm{b})=$ $0.5 \mathrm{~cm}$. Magnets lies between $-0.5 \mathrm{~cm}$ to $0.5 \mathrm{~cm}$ and $4 \mathrm{~cm}$ to $5 \mathrm{~cm}$ along $\mathrm{z}$ axis and between $-0.5 \mathrm{~cm}$ to $0.5 \mathrm{~cm}$ along $\mathrm{x}$ axis.

Fig. 12: Field visualization for repulsion of two magnets 


\section{Software for Magnetic Strength Calculation at any Desired Points}

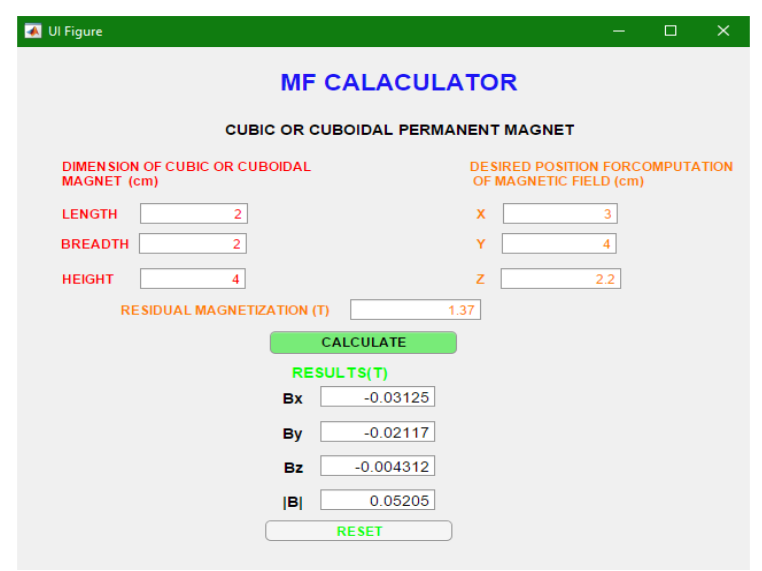

This software is applicable to cubic and cuboidal permanent magnets to compute the value of magnetic field along with the values of each components of magnetic field. It takes the input from user and displays the output on pressing the CALCULATE button. It also allows the user to reset all values of variables and result parameter to zero default value.

Fig. 13: MF Calculator for cubic or cuboidal permanent magnet

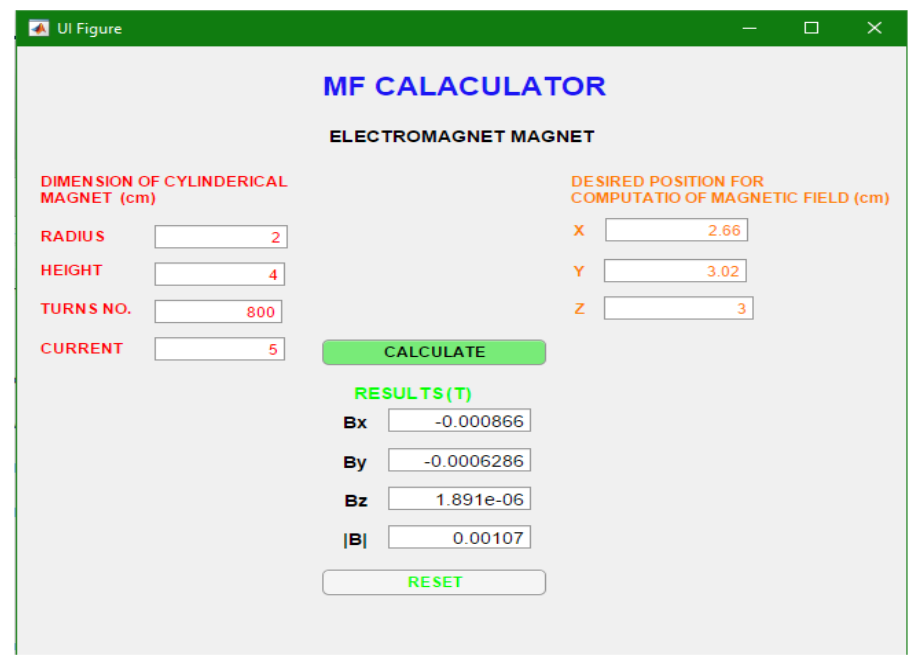

Fig. 14: MF Calculator for electromagnet magnet
This software is applicable to electromagnet to compute the value of magnetic field along with the values of each components of magnetic field. This software required input arguments from user and compute the value of magnetic field very quickly and easily.

\section{Conclusion}

This software is applicable to cylindrical permanent magnet to compute the value of magnetic field along with the values of each components of magnetic field and can be used to figure out location of magnetic vortex. It asks input arguments from user and compute the value of magnetic field. During the computation of magnetic field, the concept of shape transformation is used. The obtained value from the relation has maximum error of $1.25 \%$, when the dimension of magnet is taken to be $1 \mathrm{~cm}$ radius. The software is handy and can be transformed into Android and IOS apps. It provides user -friendly environment and enable one to solve their problems 
related to this scope easily. Field Visualization can be done by using MUPAD and values of magnetic field for each co-ordinate could be generated form the MATLAB coding. MATLAB is very powerful mathematical and Engineering tool. Application of this formula will enrich the doers with higher degree of freedom in acquisition, manipulation and storage of data for future use.

\section{References}

[1] Camacho JM and Sosa V (2013), Alternative method to calculate the magnetic field of permanent magnets with azimuthal symmetry, Revista Mexicana de F'ısica E, 59 : 8-7.

[2] Cheng DK (1983), Field and Wave Electromagnetics, Tshinghua University Press.

[3] Furlanis EP (2001), Permanent Magnet and Electromechanical device, Academic Press.

[4] Garrity TA (2015), Electricity and Magnetism for Mathematicians, Cambridge University Press.

[5] Herbert RE and Hesjedal $\mathrm{T}$ (2005), Calculation of the magnetic stray field of a uniaxial magnetic domain, Journal of Applied Physics 97(7) : 074504(1-4), DOI: 10.1063/1.1883308

[6] Lorrain P, Corson DR and Lorrain F (1987), Electromagnetic Field and Waves. W H Freeman $\&$ Co.

[7] Östman A and Ivedal M (2014), Demagnetization Effects of Assembling Halbach Arrays. Uppsala Universitet.

[8] Purcell EM, Morin DJ (2013), Electricity and Magnetism, Cambridge University Press.

[9] Ravaud R, Lemarquand G, Lemarquand V and Depollier C (2008), Analytical calculation of the magnetic field created by permanent-magnet rings, IEEE transaction on magnetics, 44(8):1982-1989.

[10] Selvaggi J, Salon S, Kwon OM and Chari MVK (2004), Calculating the External Magnetic Field from Permanent Magnets in Permanent-Magnet Motors - An Alternative Method, IEEE transactions on magnetics, 40(5) : 3278-3285.

[11] Thide B (2008), Electromagnetic Field Theory, Upsilon books, Uppsala, Sweden. 